\title{
Collisional depolarization of the lines of complex atoms/ions by neutral hydrogen
}

\author{
M. Derouich ${ }^{1}$, S. Sahal-Bréchot ${ }^{1}$, and P. S. Barklem ${ }^{2}$ \\ 1 Observatoire de Paris-Meudon, LERMA UMR CNRS 8112, 5 place Jules Janssen, 92195 Meudon Cedex, France \\ 2 Department of Astronomy and Space Physics, Uppsala University, Box 515, 75120 Uppsala, Sweden \\ e-mail: moncef.derouich@obspm.fr
}

Received 16 October 2004 / Accepted 21 December 2004

\begin{abstract}
We have developed a new semi-classical method for calculating the depolarization and polarization transfer rates of spectral lines of neutral and ionised atoms by collisions with atomic hydrogen (Derouich et al. 2003a, A\&A, 404, 763; 2003b, A\&A, 409, 369; 2004a, A\&A, 414, 369; 2004b, A\&A, 426, 707). Up to now, our depolarization rate calculations have been limited to simple atoms. In the present paper, we extend our theory to more complex atoms and we apply our new results to provide the first calculations associated with the upper levels of multiplet 145 of neutral titanium Ti I which constitutes a remarkable example of the second solar spectrum (Manso Sainz \& Landi Degl'Innocenti 2002, A\&A, 394, 1093).
\end{abstract}

Key words. Sun: atmosphere - atomic processes - line: formation - polarization - atomic data

\section{Introduction}

The so-called "second solar spectrum" is the spectrum of the linear polarization of the absorption lines observed near the limb of the Sun. This linear polarization is due to anisotropic scattering of the incident solar radiation field, the anisotropy arising from center to limb darkening.

A complete quantitative interpretation of the second solar spectrum of neutral and ionised atoms can provide accurate knowledge of the physical conditions of the solar atmospheric regions. In particular, as the magnetic field acts as a depolarizing mechanism (Hanlé effect), the comparison between the linear polarization calculated in the absence of a magnetic field and the observed polarization permits the determination of the magnetic field strength. This is currently the only tool for measuring the weak magnetic field at the surface of the quiet Sun. The physical interpretation of the polarized spectrum of the Sun requires solving the coupled sets of statistical equilibrium and radiative transfer equations taking into account all processes affecting the polarized line formation: collisions, magnetic field effects and radiative processes (see e.g. Trujillo Bueno 2003). This is a complicated problem from the theoretical and modelling viewpoints. So far, the work performed on the second solar spectrum has often been concerned with a single feature. Our understanding of this spectrum is still very limited. For the whole spectrum, more than $85 \%$ of polarized lines are emitted by complex atoms. The interpretation of the second solar spectrum raises with the problem of the interpretation of lines associated with atoms like Fe I, Ti I, Cr I, etc., and ions like Ce II, Nd II, Eu II, Ba II, Zr II, etc. For example, among neutral atomic lines reported by Gandorfer (2000), 38\% are polarized Fe I lines and $13 \%$ are from Ti I. These atoms/ions can suffer isotropic collisions with hydrogen atoms before they radiate. To evaluate the effect of these collisions, methods capable of giving realistic results for many levels of atoms or ions and which are computationally efficient are urgently needed. In fact, it is very difficult and sometimes impossible to treat collision processes involving neutral and ionised heavy atoms by standard quantum chemistry methods. The depolarizing rates of complex atoms have not been obtained by any theoretical method (at least to our knowledge). In the last few years, we have developed a general semi-classical theory which allows us to compute in a fairly realistic way collisional depolarization rates for all transitions of simple atoms (Derouich et al. 2003a,b, 2004a,b, hereafter Papers I, II, III and IV respectively). We thus can obtain the depolarization rates for any level of any simple atom by interpolation of our results given in the form of tables.

The goal of the present paper is to generalize our method to complex atoms. The Grotrian diagram for complex atoms can have a complicated structure, with different possible terms ${ }^{2 S+1} L_{J}$ for which depolarization rates cannot be derived by extrapolation or interpolation of our results of Papers I, II, III and IV. We recall that we assumed that the interaction potential is dominated by the optical electron, and then it is only the optical electron that can undergo transitions between Zeeman sublevels during the collision (the frozen core approximation); this does not imply that the ionic core is a closed-shell. Using the frozen core approximation and the fact that the fine structure can be neglected during the collision, we will express the 
scattering matrix elements of complex atoms colliding with hydrogen atoms as a linear combination of those of simple atoms colliding with hydrogen atoms. Given this generalization of our semi-classical method, it will now be possible to obtain results for complex atoms/ions with reasonable accuracy; we expect an accuracy better than $20 \%$. With our semi-classical method we are able to compute the needed rates for all levels very quickly.

\section{Statement of the problem}

We consider a perturbed atom/ion in $L S$ coupling with an electronic configuration composed of three parts:

1. electrons in a filled subshell, that is a configuration containing the maximum number of equivalent electrons (having the same value of $n$ and $l$ ); there is only one possible spectral term, namely the term ${ }^{1} \mathrm{~S}_{0}$;

2. equivalent electrons in an incomplete (open) subshell. We denote by $\boldsymbol{L}_{\boldsymbol{c}}$ the total orbital angular momentum of these electrons and by $\boldsymbol{S}_{\boldsymbol{c}}$ their total spin. ${ }^{2 S_{c}+1} L_{c}$ is the so-called parent term. We recall that for a given parent configuration of the equivalent electrons in a partly-filled subshell there arises more than one parent term: in this case the parent term is written in parentheses after the parent configuration;

3 . the optical electron with orbital angular momentum $\boldsymbol{l}$ and spin $s(s=1 / 2)$. Therefore, we limit ourselves to the case where there is only one electron in the external shell. For example, the $4 \mathrm{f}^{7}\left({ }^{8} \mathrm{~S}\right) 6 \mathrm{~s} 6 \mathrm{p}^{2}\left({ }^{4} \mathrm{P}\right){ }^{11} \mathrm{P}_{5}$ level is outside the scope of the present paper but levels like $3 d^{7}\left({ }^{4} F\right) 4 p\left({ }^{5} G_{4}\right)$ and $3 d^{7}\left({ }^{4} \mathrm{~F}\right) 4 \mathrm{~d}\left({ }^{5} \mathrm{H}_{5}\right)$ levels can be treated as will be shown in Sect. 5.

We denote by $\boldsymbol{L}=\boldsymbol{L}_{\boldsymbol{c}}+\boldsymbol{l}$ the total orbital momentum of the perturbed atom, by $\boldsymbol{S}=\boldsymbol{S}_{\boldsymbol{c}}+\boldsymbol{s}$ the total spin and by $\boldsymbol{J}=\boldsymbol{L}+\boldsymbol{S}$ the total angular momentum. For a given configuration there are several possible terms ${ }^{2 S+1} L_{J}$ (that is, the possible values of $L$ and $S$ ) built on each of the parent terms of the equivalent electrons from incomplete subshells and adding to them the spin and the orbital angular momentum of the valence electron. One must reject the values of $L$ and $S$ corresponding to states forbidden by the Pauli exclusion principle.

In our collision problem, the complex atomic/ionic species, like Ti I, Fe I, Ce II, etc, collides with a bath of perturbing hydrogen atoms. In the solar photosphere and the low chromosphere conditions, the hydrogen atom can be assumed to remain in its ground state during the collision. We denote by $\left|n l m_{l}\right\rangle$ the state of the valence electron of the perturbed atom/ion in the atomic frame in which the perturbed atom/ion is stationary at the origin and the axis of quantization is taken perpendicular to the collision plane. The linear transformation connecting the two representations $\left|l m_{l}\right\rangle\left|L_{c} M_{L_{c}}\right\rangle$ and $\left|L M_{L}\right\rangle$ is

$\left|L M_{L}\right\rangle=\sum_{m_{l}, M_{L_{c}}} C_{M_{L_{c}} m_{l} M_{L}}^{L_{c} l L}\left|L_{c} M_{L_{c}}\right\rangle\left|l m_{l}\right\rangle$,

where $C_{M_{L} m_{l} M_{L}}^{L_{c} l L}$ is a Clebsch-Gordan coefficient given by (Messiah 1961):

$C_{M_{L_{c}} m_{l} M_{L}}^{L_{c} L}=\sqrt{2 L+1}(-1)^{L_{c}-l+M_{L}}\left(\begin{array}{ccc}L_{c} & l & L \\ M_{L_{c}} & m_{l} & -M_{L}\end{array}\right)$.
The expression between parentheses denotes a $3 j$-coefficient. The scattering matrix elements

$$
\begin{aligned}
\left\langle L M_{L}\left|S_{\mathrm{sc}}\right| L^{\prime} M_{L}^{\prime}\right\rangle= & \sum_{m_{l}, M_{L_{c}}, m_{l}^{\prime}, M_{L_{c}}^{\prime}} C_{M_{L_{c}} m_{l} M_{L}}^{L_{c} l L} C_{M_{L_{c}}^{\prime} m_{l}^{\prime} M_{L}^{\prime}}^{L_{c}^{\prime} l^{\prime}} \\
& \times\left\langle l m_{l}\left|\left\langle L_{c} M_{L_{c}}\left|S_{\mathrm{sc}}\right| L_{c}^{\prime} M_{L_{c}}^{\prime}\right\rangle\right| l^{\prime} m_{l}^{\prime}\right\rangle .
\end{aligned}
$$

We assume that only the optical electron is affected by the interaction with neutral hydrogen. The collision does not affect the state of internal electrons such that $\boldsymbol{L}_{\boldsymbol{c}}, \boldsymbol{S}_{\boldsymbol{c}}$ and $\boldsymbol{J}_{\boldsymbol{c}}$ are conserved. This assumption has been adopted in our previous Papers (I, II, III and IV) and in the ABO papers (Anstee 1992; Anstee \& O'Mara 1991, 1995; Anstee et al. 1997; Barklem 1998; Barklem \& O’Mara 1997; Barklem et al. 1998).

Consequently, the scattering matrix is diagonal in $L_{c}$ and its elements do not depend on $M_{L_{c}}$. Thus $M_{L_{c}}^{\prime}=M_{L_{c}}$, and

$$
\begin{aligned}
\left\langle n L M_{L}\left|S_{\mathrm{sc}}\right| n L^{\prime} M_{L}^{\prime}\right\rangle= & \sum_{\substack{m_{l}, m_{l}^{\prime}, M_{L_{c}}\\
}} C_{M_{L_{c}} m_{l} M_{L}}^{L_{c} l L} C_{M_{L_{c}} m_{l}^{\prime} M_{L}^{\prime}}^{L_{l}{ }^{\prime} L^{\prime}} \\
& \times\left\langle n l m_{l}\left|S_{\mathrm{sc}}\right| n l^{\prime} m_{l}^{\prime}\right\rangle .
\end{aligned}
$$

In Eq. (4), we consider only the case where $l=l^{\prime}$ since quenching is neglected. The scattering matrix elements $\left\langle n l m_{l}\left|S_{\text {sc }}\right| n l m_{l}^{\prime}\right\rangle$ are obtained after integration of the semi-classical differential coupled equations which are derived from the timedependent Schrödinger equation (Papers I, II, III and IV) followed by the calculation of the scattering matrix elements $\left\langle n L M_{L}\left|S_{\text {sc }}\right| n L^{\prime} M_{L}^{\prime}\right\rangle$ using Eq. (4).

In order to determine the depolarization and polarization transfer rates of the term ${ }^{2 S+1} L_{J}$, we need the $S_{\mathrm{sc}}$-matrix elements in the $\left|n J M_{J}\right\rangle$ basis, so we need to express the scattering matrix elements $\left\langle n J M_{J}\left|S_{\mathrm{sc}}\right| n J^{\prime} M_{J}^{\prime}\right\rangle$ as a function of the $\left\langle n L M_{L}\left|S_{\text {sc }}\right| n L M_{L}^{\prime}\right\rangle$ elements. We can show that

$$
\begin{aligned}
& \left\langle n J M_{J}\left|S_{\mathrm{sc}}\right| n J^{\prime} M_{J}^{\prime}\right\rangle=\sum_{M_{L}, M_{S}, M_{L}^{\prime}, M_{S}^{\prime}} C_{M_{L} M_{S} M_{J}}^{L S J} \\
& \quad \times C_{M_{L}^{\prime} M_{S}^{\prime} J^{\prime} M_{J}^{\prime}}\left\langle n L M_{L}\left|\left\langle n S M_{S}\left|S_{\mathrm{sc}}\right| n S^{\prime} M_{S}^{\prime}\right\rangle\right| n L^{\prime} M_{L}^{\prime}\right\rangle .
\end{aligned}
$$

The spin is neglected during the collision, thus the $S_{\mathrm{sc}}$-matrix is diagonal in $S$ and its elements do not depend on $M_{S}$ (the vector $S$ is conserved), therefore $S=S^{\prime}, M_{S}=M_{S}^{\prime}$ and

$$
\begin{aligned}
\left\langle n J M_{J}\left|S_{\mathrm{sc}}\right| n J^{\prime} M_{J}^{\prime}\right\rangle= & \sum_{M_{L}, M_{S}, M_{L}^{\prime}} C_{M_{L} M_{S} M_{J}}^{L S J} C_{M_{L}^{\prime} M_{S} M_{J}^{\prime}}^{L S J^{\prime}} \\
& \times\left\langle n L M_{L}\left|S_{\mathrm{sc}}\right| n L^{\prime} M_{l}^{\prime}\right\rangle
\end{aligned}
$$

which gives, using the expression for $\left\langle n L M_{L}|S| n L^{\prime} M_{L}^{\prime}\right\rangle$ given by Eq. (4) and after some angular algebra transformations,

$$
\begin{aligned}
& \left\langle n J M_{J}\left|S_{\mathrm{sc}}\right| n J^{\prime} M_{J}^{\prime}\right\rangle=(-1)^{J+J^{\prime}+M_{J}+M_{J}^{\prime}} \\
& \times \sqrt{(2 J+1)\left(2 J^{\prime}+1\right)} \sqrt{(2 L+1)\left(2 L^{\prime}+1\right)} \\
& \times \sum_{J^{\prime \prime}}\left(2 J^{\prime \prime}+1\right)\left\{\begin{array}{ccc}
L_{c} & L & l \\
L^{\prime} & S & J^{\prime} \\
l & J & J^{\prime \prime}
\end{array}\right\} \\
& \times \sum_{m_{l}, m_{l}^{\prime}, M_{J}^{\prime \prime}}\left\langle n l m_{l}\left|S_{\mathrm{sc}}\right| n l m_{l}^{\prime}\right\rangle\left(\begin{array}{ccc}
l & J^{\prime} & J^{\prime \prime} \\
m_{l}^{\prime} M_{J}^{\prime} & M_{J}^{\prime \prime}
\end{array}\right) \\
& \times\left(\begin{array}{ccc}
l & J & J^{\prime \prime} \\
m_{l} & M_{J} & M_{J}^{\prime \prime}
\end{array}\right)
\end{aligned}
$$




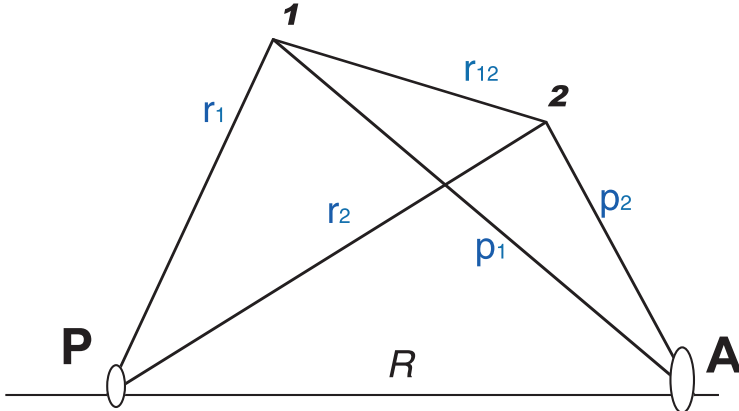

Fig. 1. Only the optical electron of the complex atom $A$, denoted by 2 , interacts with neutral hydrogen core (a proton) located at $P$ and its valence electrons denoted by 1 . The collision does not affect the other internal electrons in filled or in incomplete subshells.

where \{\} ( 3 lines $\times 3$ columns) denotes a Wigner $9 j$-coefficient (Messiah 1961). Equation (7) shows that, in the approximations described above, $\left\langle n J M_{J}|S| n J^{\prime} M_{J}^{\prime}\right\rangle$ can be written as a linear combination of the $\left\langle n l m_{l}|S| n l m_{l}^{\prime}\right\rangle$ and so the interaction potential needed to calculate the scattering matrix $S_{\text {sc }}$ between the sublevels $\left|n J M_{J}\right\rangle \rightarrow\left|n J^{\prime} M_{J}^{\prime}\right\rangle\left(J=J^{\prime}\right.$ and $\left.J \neq J^{\prime}\right)$ is the one needed to calculate $\left\langle n l m_{l}|S| n l m_{l}^{\prime}\right\rangle$. This is the most important conclusion of the present work.

\section{Interaction potential}

The model for the interaction between the hydrogen perturber and the perturbed complex atom/ion, which is assumed to be totally electrostatic, is shown in Fig. 1. The interaction potential $V$ has a cylindrical symmetry about the interatomic axis and so it is diagonal in the rotating frame which is in fact more convenient for the interaction potential calculation (Roueff 1974). In this frame, we denote by $\mu_{l}$ the projection of the orbital angular momentum of the valence electron $l$ on to the interatomic axis which is taken as the axis of quantization. The $(l+1)$ elements $V_{\mathrm{eff}, \mu_{l}}\left(0 \leq \mu_{l} \leq l\right)$ of the so-called RSU interaction potential matrix are given by (more details in the ABO papers, see also Papers I, II, III, IV):

$$
\begin{aligned}
V_{\mathrm{eff}, \mu_{l}}= & V_{\mathrm{eff},-\mu_{l}}=\left\langle 100\left|\left\langle n l \mu_{l}\left|V_{\mathrm{eff}}\right| n l \mu_{l}\right\rangle\right| 100\right\rangle \\
= & \left\langle 100\left|\left\langle n l \mu_{l}|V| n l \mu_{l}\right\rangle\right| 100\right\rangle \\
& -\frac{1}{E_{p}}\left(\left\langle 100\left|\left\langle n l \mu_{l}|V| n l \mu_{l}\right\rangle\right| 100\right\rangle\right)^{2} \\
& +\frac{1}{E_{p}} \int_{0}^{+\infty} P_{n^{*} l}^{2} I_{l \mu_{l}}\left(R, p_{2}\right) \mathrm{d} p_{2},
\end{aligned}
$$

where $I_{l \mu_{l}}$ are lengthy complicated analytic functions (Anstee 1992; Barklem 1998) and $E_{p}$ is the Unsöld energy (Unsöld 1927, 1955; ABO papers; see also Papers I, II, III and IV for more details about $E_{p}$ according to depolarizing collisions). $P_{n^{*} l}$ is the Coulomb radial wave function for the valence electron of the perturbed atom with quantum defect $\delta=n-n^{*}$.

The interaction potentials enter into the semi-classical coupled differential equations arising from the Schrödinger equation. These coupled equations must be solved to obtain the $(2 l+1)^{2} S_{\mathrm{sc}}$-matrix elements $\left\langle n l m_{l}\left|S_{\mathrm{sc}}\right| n l m_{l}^{\prime}\right\rangle$. For a given quantum number $l$ we obtain $(2 l+1)$ coupled differential equations.

\section{Depolarization and polarization transfer rates}

Using Eq. (7), we obtain $\left\langle n L J M_{J}\left|S_{\mathrm{sc}}(\boldsymbol{b}, \boldsymbol{v})\right| n L J M_{J}^{\prime}\right\rangle$ in the case where the quantization axis is taken as perpendicular to the collision plane $(\boldsymbol{b}, \boldsymbol{v})$. The depolarizing collisions are isotropic and we now have to perform an average over all possible orientations describing the collision plane $(\boldsymbol{b}, \boldsymbol{v})$ in order to obtain the transition probabilities

$$
\begin{aligned}
P\left(n L J M_{J}\right. & \left.\left.\rightarrow n L J M_{J}^{\prime}, b, v\right)\right\rangle_{\mathrm{av}}= \\
& \frac{1}{8 \pi^{2}} \oint \mathrm{d} \Omega\left|\left\langle n L J M_{J}\left|I-S_{\mathrm{sc}}(\boldsymbol{b}, \boldsymbol{v})\right| n L J M_{J}^{\prime}\right\rangle\right|^{2},
\end{aligned}
$$

where $I$ is the unit matrix and $T(\boldsymbol{b}, \boldsymbol{v})=I-S_{\mathrm{sc}}(\boldsymbol{b}, \boldsymbol{v})$ is the collisional transition matrix between the Zeeman sublevels depending on the impact-parameter $\boldsymbol{b}$ and relative velocity $\boldsymbol{v}$.

The statistical equilibrium equations can be expressed on the basis of irreducible tensorial operators (ITO) which is especially well adapted to the context of scattering polarization and the Hanlé effect (cf. Sahal-Bréchot 1977; Landi Degl'Innocenti 1982). The ITO are defined in Brink and Satchler (1962) or any other book devoted to angular momentum theory (e.g. Blum 1981). In the ITO representation, the average depolarization probability $\left(J=J^{\prime}\right)$ of tensorial rank $k$ is a linear combination of the transition probabilities between the Zeeman sublevels (Sahal-Bréchot 1977):

$$
\begin{aligned}
\left\langle P^{k}(n L J, b, v)\right\rangle_{\mathrm{av}}= & \langle P(n L J, b, v)\rangle_{\mathrm{av}}-(2 k+1) \\
& \times \sum_{M_{J}, M_{J}^{\prime}}(-1)^{2 J-M_{J}-M_{J}^{\prime}}\left(\begin{array}{ccc}
J & k & J \\
-M_{J} 0 M_{J}
\end{array}\right) \\
& \times\left(\begin{array}{ccc}
J & k & J \\
-M_{J}^{\prime} 0 M_{J}^{\prime}
\end{array}\right)\left\langle P\left(L J M_{J} \rightarrow L J M_{J}^{\prime}, b, v\right)\right\rangle_{\mathrm{av}},
\end{aligned}
$$

and the average collisional polarization transfer probability $\left(J \neq J^{\prime}\right)$ is given by (Sahal-Bréchot 1977):

$$
\begin{aligned}
\left\langle P^{k}(n L J\right. & \left.\left.\rightarrow n L J^{\prime}, b, v\right)\right\rangle_{\mathrm{av}}=(2 k+1) \\
& \times \sum_{M_{J}, M_{J}^{\prime}}(-1)^{J+J^{\prime}-M_{J}-M_{J}^{\prime}}\left(\begin{array}{ccc}
J & k & J \\
-M_{J} 0 M_{J}
\end{array}\right) \\
& \times\left(\begin{array}{cc}
J^{\prime} & k J^{\prime} \\
-M_{J}^{\prime} 0 M_{J}^{\prime}
\end{array}\right)\left\langle P\left(L J M_{J} \rightarrow L J^{\prime} M_{J}^{\prime}, b, v\right)\right\rangle_{\mathrm{av}}
\end{aligned}
$$

Integration of the probabilities given by the above equations over the impact-parameter $b$ and over a Maxwell distribution of velocities $f(v)$ for a temperature $T$ of the medium, can be performed to obtain the depolarization $\left(J=J^{\prime}\right)$ and the polarization transfer $\left(J \neq J^{\prime}\right)$ rates (Papers I and II):

$$
\begin{aligned}
& D^{k}\left(n L J \rightarrow n L J^{\prime}, T\right)\left(J=J^{\prime} \text { and } J \neq J^{\prime}\right) \simeq n_{\mathrm{H}} \int_{0}^{\infty} v f(v) \mathrm{d} v \\
& \times\left(\pi b_{0}^{2}+2 \pi \int_{b_{0}}^{\infty}\left\langle P^{k}\left(n L J \rightarrow n L J^{\prime}, b, v\right)\right\rangle_{\mathrm{av}} b \mathrm{~d} b\right),
\end{aligned}
$$

where $n_{\mathrm{H}}$ is the neutral hydrogen density, $b_{0}$ is the impactparameter cutoff and we use $b_{0}=3 a_{0}$ as in Papers I, II, III and IV. 


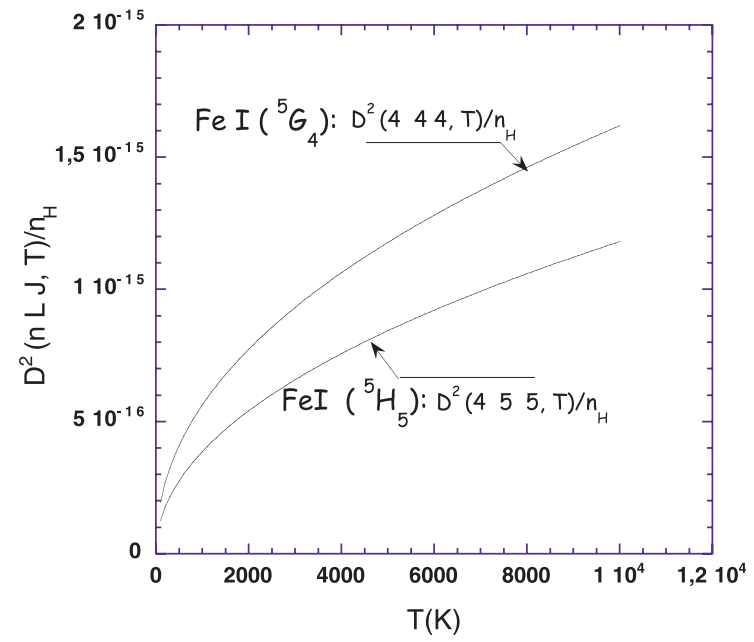

Fig. 2. Depolarization rates per unit $\mathrm{H}$-atom density for the $\mathrm{Fe} \mathrm{I}$ atom, $D^{2}(444, T) / n_{\mathrm{H}}$ and $D^{2}(455, T) / n_{\mathrm{H}}$, as a function of temperature $T$. The rates are given in $\operatorname{rad~}^{3} \mathrm{~s}^{-1}$.

\section{How does our theory work?}

To demonstrate how the theory works, we treat in detail the example of the Fe I $5396 \AA$ line which is the transition between the $3 d^{7}\left({ }^{4} F\right) 4 p\left({ }^{5} G_{4}\right)$ and $3 d^{7}\left({ }^{4} F\right) 4 d\left({ }^{5} \mathrm{H}_{5}\right)$ levels. Let us consider the collision between the iron atom in each of these two states and a bath of perturbing hydrogen atoms in their ground state $1 \mathrm{~s}\left({ }^{2} \mathrm{~S}_{1 / 2}\right)$.

1. For the level Fe $\mathbf{I}\left(3 \mathrm{~d}^{7}\left({ }^{4} \mathrm{~F}\right) 4 \mathrm{p}\left({ }^{5} \mathrm{G}_{4}\right)\right)$, we have $L_{c}=3, l=1$, $L=4, J=4$ and $S=2$. While our previous Papers (I, II, III and IV) deal only with states with angular momentum $\leq 3(p, d$, and $f)$, and the angular momentum of the state is $L=4>3$, this does not mean that the calculation of depolarization is not possible with our theory. The calculation of depolarization rates for Fe I $\left(3 d^{7}\left({ }^{4} F\right) 4 p\left({ }^{5} G_{4}\right)\right)$ in fact derives from our results obtained for the $p$ state (Paper I). This is because the orbital angular momentum of the optical electron, which is in $4 \mathrm{p}$, is $l=1$. Therefore, the scattering matrix elements $\left\langle 44 M_{J}\left|S_{\mathrm{sc}}\right| 44 M_{J}^{\prime}\right\rangle$ are given by a linear combination of the scattering matrix elements $\left\langle 41 m_{l}\left|S_{\text {sc }}\right| 41 m_{l}^{\prime}\right\rangle$ as in Eq. (7). The destruction rate of alignment of the level $3 \mathrm{~d}^{7}\left({ }^{4} F\right) 4 \mathrm{p}\left({ }^{5} G_{4}\right)$ for the local temperature $T, D^{2}(444, T)$, is calculated as explained in Sect. 4 and we find the following analytical expression in $\mathrm{rad} \mathrm{s}^{-1}$ for $100 \leq T \leq 10000 \mathrm{~K}$ (see Fig. 2):

$$
D^{2}(444, T)=1.1774 \times 10^{-15} n_{\mathrm{H}}\left(\frac{T}{5000}\right)^{0.459} .
$$

2. In the case of the upper level of the Fe I line, $3 d^{7}\left({ }^{4} F\right) 4 d$ $\left({ }^{5} \mathrm{H}_{5}\right), L_{c}=3, l=2, L=5, J=5$ and $S=2$. The scattering matrix elements $\left\langle 45 M_{J}\left|S_{\text {sc }}\right| 45 M_{J}^{\prime}\right\rangle$ can be written as a linear combination of the scattering matrix elements $\left\langle 42 m_{l}\left|S_{\text {sc }}\right| 42 m_{l}^{\prime}\right\rangle$. We thus can obtain the destruction rate of alignment of the level $3 \mathrm{~d}^{7}\left({ }^{4} \mathrm{~F}\right) 4 \mathrm{~d}\left({ }^{5} \mathrm{H}_{5}\right)$ in $\mathrm{rad} \mathrm{s}^{-1}$ $(100 \leq T \leq 10000 \mathrm{~K})$ (see Fig. 2),

$D^{2}(455, T)=0.8430 \times 10^{-15} n_{\mathrm{H}}\left(\frac{T}{5000}\right)^{0.485}$.
We do not give explicit expressions for $\left\langle 44 M_{J}\left|S_{\mathrm{sc}}\right| 44 M_{J}^{\prime}\right\rangle$ and $\left\langle 45 M_{J}\left|S_{\mathrm{sc}}\right| 45 M_{J}^{\prime}\right\rangle$ according to Eq. (7), as they are rather lengthy, consisting of $(2 J+1)^{2}$ matrix elements. The linear combination coefficients may be obtained on request from the authors.

\section{Application}

The polarimetric observations at the limb of the Sun show that one of the more interesting features is the linear polarization of lines associated with multiplet 145 of the Ti I atom (Manso Sainz \& Landi Degl'Innocenti 2002). Since $L=2$ and $S=2$ in the upper term level $w^{5} D$ of multiplet 145 of Ti I, $J$ takes on the values $0,1,2,3$ and 4 . The destruction rate of alignment of the state ${ }^{5} \mathrm{D}_{0}$ is necessarily zero because this state cannot be aligned by definition $(J=0)$. In the case of the level $3 \mathrm{~d}^{3}\left({ }^{4} \mathrm{P}\right) 4 \mathrm{p}\left({ }^{5} \mathrm{D}_{1}\right), L_{c}=1, l=1, L=2, J=1$ and $S=2$. According to Eq. (7), the nine $(J=1)$ matrix elements $\left\langle n 1 M_{J}\left|S_{\mathrm{sc}}\right| n 1 M_{J}^{\prime}\right\rangle$ are given as function of the $\left\langle n 1 m_{l}\left|S_{\mathrm{sc}}\right| n 1 m_{l}^{\prime}\right\rangle$ by:

$$
\begin{aligned}
\left\langle 1-1\left|S_{\mathrm{sc}}\right| 1-1\right\rangle= & \frac{3}{20}\left\langle 11\left|S_{\mathrm{sc}}\right| 11\right\rangle+\frac{9}{20}\left\langle 10\left|S_{\mathrm{sc}}\right| 10\right\rangle \\
& +\frac{8}{20}\left\langle 1-1\left|S_{\mathrm{sc}}\right| 1-1\right\rangle, \\
\left\langle 1-1\left|S_{\mathrm{sc}}\right| 10\right\rangle= & -\frac{3}{10}\left\langle 11\left|S_{\mathrm{sc}}\right| 10\right\rangle+\frac{1}{20}\left\langle 10\left|S_{\mathrm{sc}}\right| 1-1\right\rangle, \\
\left\langle 1-1\left|S_{\mathrm{sc}}\right| 11\right\rangle= & -\frac{7}{20}\left\langle 11\left|S_{\mathrm{sc}}\right| 1-1\right\rangle, \\
\left\langle 10\left|S_{\mathrm{sc}}\right| 1-1\right\rangle= & -\frac{3}{10}\left\langle 10\left|S_{\mathrm{sc}}\right| 11\right\rangle+\frac{1}{20}\left\langle 1-1\left|S_{\mathrm{sc}}\right| 10\right\rangle, \\
\left\langle 10\left|S_{\mathrm{sc}}\right| 10\right\rangle= & \frac{1}{10}\left\langle 10\left|S_{\mathrm{sc}}\right| 10\right\rangle+\frac{9}{20}\left\langle 11\left|S_{\mathrm{sc}}\right| 11\right\rangle \\
& +\frac{9}{20}\left\langle 1-1\left|S_{\mathrm{sc}}\right| 1-1\right\rangle, \\
\left\langle 10\left|S_{\mathrm{sc}}\right| 11\right\rangle= & \frac{1}{20}\left\langle 11\left|S_{\mathrm{sc}}\right| 10\right\rangle-\frac{3}{10}\left\langle 10\left|S_{\mathrm{sc}}\right| 1-1\right\rangle, \\
\left\langle 11\left|S_{\mathrm{sc}}\right| 1-1\right\rangle= & -\frac{7}{20}\left\langle 1-1\left|S_{\mathrm{sc}}\right| 11\right\rangle, \\
\left\langle 11\left|S_{\mathrm{sc}}\right| 10\right\rangle= & \frac{1}{20}\left\langle 10\left|S_{\mathrm{sc}}\right| 11\right\rangle-\frac{3}{10}\left\langle 1-1\left|S_{\mathrm{sc}}\right| 10\right\rangle, \\
\left\langle 11\left|S_{\mathrm{sc}}\right| 11\right\rangle= & \frac{2}{5}\left\langle 11\left|S_{\mathrm{sc}}\right| 11\right\rangle+\frac{9}{20}\left\langle 10\left|S_{\mathrm{sc}}\right| 10\right\rangle \\
& \left.+1\left|S_{\mathrm{sc}}\right| 1-1\right\rangle . \\
\langle &
\end{aligned}
$$

Having $\left\langle 1 M_{J}\left|S_{\mathrm{sc}}\right| 1 M_{J}^{\prime}\right\rangle$, we then computed the depolarization rate $D^{2}(421, T)$ as defined in Sect. 4 and the analytical expression in $\mathrm{rad} \mathrm{s}^{-1}$ for $100 \leq T \leq 10000 \mathrm{~K}$ is

Ti I $\left(3 d^{3}\left({ }^{4} \mathrm{P}\right) 4 \mathrm{p}\left({ }^{5} \mathrm{D}_{1}\right)\right)-\mathbf{H}(1 \mathrm{~s})$ :

$D^{2}(421, T)=2.5126 \times 10^{-15} n_{\mathrm{H}}\left(\frac{T}{5000}\right)^{0.412}$,

and by using the same procedure for determining the depolarization rates of the sublevels ${ }^{5} \mathrm{D}_{2,3,4}$, we obtain in $\operatorname{rad~s}{ }^{-1}$ 


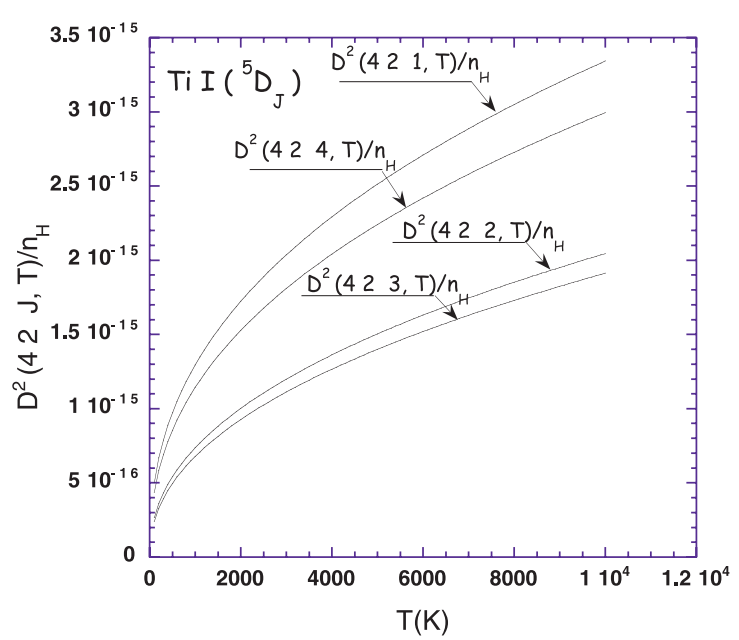

Fig. 3. Depolarization rates per unit $\mathrm{H}$-atom density for the upper levels of multiplet 145 of the Ti I atom, $D^{2}(42 J, T) / n_{\mathrm{H}}$, as a function of temperature $T . D^{2}(44 J, T) / n_{\mathrm{H}}$ is given in $\mathrm{rad} \mathrm{m}^{3} \mathrm{~s}^{-1}$.

$(100 \leq T \leq 10000 \mathrm{~K})$.

Ti I $\left(3 d^{3}\left({ }^{4} P\right) 4 p\left({ }^{5} D_{2}\right)\right)-\mathbf{H}(1 s)$ :

$D^{2}(422, T)=1.5036 \times 10^{-15} n_{\mathrm{H}}\left(\frac{T}{5000}\right)^{0.443} ;$

Ti I $\left(3 d^{3}\left({ }^{4} \mathrm{P}\right) 4 p\left({ }^{5} D_{3}\right)\right)-\mathbf{H}(1 s)$ :

$D^{2}(423, T)=1.3999 \times 10^{-15} n_{\mathrm{H}}\left(\frac{T}{5000}\right)^{0.450} ;$

and $\mathbf{T i} \mathbf{I}\left(3 \mathrm{~d}^{3}\left({ }^{4} \mathrm{P}\right) 4 \mathrm{p}\left({ }^{5} \mathrm{D}_{4}\right)\right)-\mathbf{H}(1 \mathrm{~s})$ :

$D^{2}(424, T)=2.2410 \times 10^{-15} n_{\mathrm{H}}\left(\frac{T}{5000}\right)^{0.420}$.

The results for the destruction rate of alignment of the excited states ${ }^{5} \mathrm{D}_{\mathrm{J}}$ are given in Fig. 3.

We notice that the effective principal quantum number corresponding to the state of the valence electron $4 \mathrm{p}$ is $n^{*}=2.37$. The average depolarization rate at $T=$ $5000 \mathrm{~K}$, per unit $\mathrm{H}$-atom density, of the term levels ${ }^{5} \mathrm{D}_{1,2,3,4}$ is $\sim 1.9 \times 10^{-15}\left(\mathrm{rad} \mathrm{s}^{-1}\right)$. However, by interpolation of Table 4 of Paper I, giving the variation of the depolarization rate at $5000 \mathrm{~K}$ with effective principal quantum number, one obtains for $n^{*}=2.37$ that the depolarization rate per unit $\mathrm{H}$-atom density is $18.42 \times 10^{-15}\left(\mathrm{rad} \mathrm{s}^{-1}\right)$. The depolarization rates for the multiplet of Ti I are about a factor of ten less than depolarization rates for the corresponding case of simple atoms. This is due to the effect of equivalent electrons in an incomplete subshell having non-zero total orbital angular momentum $L_{c}$. Under similar conditions in the solar photosphere, the atomic polarization of simple atoms/ions having $L_{c}=0$ is more strongly affected by isotropic collisions with neutral hydrogen than the atomic polarization of complex atoms/ions (Fe I, Ti I, Cr I, Ce II, Nd II, ...). Coupled with other factors, this helps explain why the majority $(85 \%)$ of the polarized lines of the second solar spectrum are emitted by complex atoms/ions.

We consider only the collisional polarization transfer $D^{k}\left(n L J \rightarrow n L J^{\prime}, T\right)$ pertaining to the $|n L\rangle$ level. The atomic

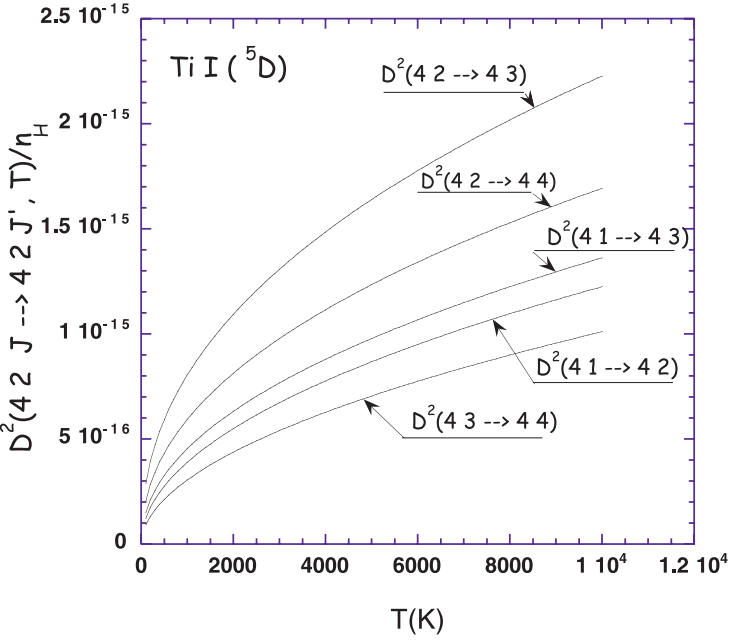

Fig. 4. Polarization transfer rates per unit $\mathrm{H}$-atom density for the upper level of the multiplet $145, D^{k}\left(42 J \rightarrow 42 J^{\prime}, T\right) / n_{\mathrm{H}}$, as a function of $T$. The rates are given in $\operatorname{rad~}^{3} \mathrm{~s}^{-1}$.

linear polarization transfer rates $(k=2) D^{2}\left(n L J \rightarrow n L J^{\prime}, T\right)$ as a function of $T$ are displayed in Fig. 4. We can provide analytical expressions, in $\mathrm{rad} \mathrm{s}^{-1}$, for the non-zero transfer rates:

$$
\begin{aligned}
& D^{2}(421 \rightarrow 422, T)=8.6724 \times 10^{-16} n_{\mathrm{H}}\left(\frac{T}{5000}\right)^{0.498}, \\
& D^{2}(421 \rightarrow 423, T)=9.7806 \times 10^{-16} n_{\mathrm{H}}\left(\frac{T}{5000}\right)^{0.478}, \\
& D^{2}(422 \rightarrow 423, T)=16.3915 \times 10^{-16} n_{\mathrm{H}}\left(\frac{T}{5000}\right)^{0.442}, \\
& D^{2}(422 \rightarrow 424, T)=12.3407 \times 10^{-16} n_{\mathrm{H}}\left(\frac{T}{5000}\right)^{0.456}, \\
& D^{2}(423 \rightarrow 424, T)=7.0415 \times 10^{-16} n_{\mathrm{H}}\left(\frac{T}{5000}\right)^{0.521}
\end{aligned}
$$

All rates were found to increase with temperature $T$. As for the case of simple atoms, the functional form $D(T)=A T^{(1-\lambda) / 2}$, where $\lambda$ is the so-called velocity exponent, may be accurately fitted to the depolarization rates and the population transfer rates.

\section{Discussion}

Unfortunately, there are neither experimental nor quantum chemistry depolarization and collisional transfer rates for complex atoms with which to compare. To generalize our method to the complex atoms, we do not assume any further approximation with respect to those adopted in our previous Papers (I, II, III, IV). We therefore expect the error in the depolarization rates of complex atoms to be similar to that for the rates of simple atoms, i.e. less than $20 \%$. At the intermediate to longrange interatomic distances, the interaction energy between the two atoms is smaller than the energy eigenvalues of the isolated atoms, so that perturbation theory is suitable for calculating the RSU interatomic potentials used in this work. The depolarization and polarization transfer rates are due essentially to intermediate-range interactions (Paper I). In fact, we have 
found that a mutiplication of the potential values by a factor of two, at the close or long-range regions of interaction, practically does not change the values of the calculated probabilities. The main differences between the semi-classical potentials used here and those from a sophisticated quantum chemistry approach occur at small interatomic separations. Since these close regions do not influence the values of depolarization rates, the differences with quantum chemistry results are usually less than $20 \%$. Derouich (2004) has shown that an error of $20 \%$, which represents the maximum error expected in our rates, is sufficient to determine the magnetic field by interpretation of the Hanlé effect with a satisfactory precision. For instance, in the case of the SrI $4607 \AA$ line, we have shown that an inaccurarcy of $20 \%$ on the depolarization rates leads to a $6 \%$ inaccurarcy only on the magnetic field determination (see also Bommier et al. 2004).

Finally we note that, contrary to what is sometimes employed in astrophysics, the depolarization rates are not proportional to the collisional broadening rates, in particular the depolarization rate is not equal to one half the collisional broadening rate. For example, using the ABO theory, the collisional broadening rate per unit $\mathrm{H}$-atom density for the $\mathrm{p}-\mathrm{d}$ transition corresponding to the Fe I $5396 \AA$ line is $\sim 3.3 \times 10^{-14} \mathrm{rad} \mathrm{s}^{-1}$ at $5000 \mathrm{~K}$, that is to say $\sim 30$ times greater than the depolarization rates associated to this line, as given by Eqs. (13) and (14). The method, which has been used until now due to the lack of any better technique, consisting of obtaining the depolarization rates using Van der Waals collisional broadening rates, can lead to very inaccurate results. The error in the depolarization rates inferred from this approximation can be much more important than the error in the collisional broadening rates from the use of the classical Van der Waals potential formula, which gives rates typically underestimated by factors of two or more. In other words, for these depolarization rates, the error mainly arises both from the use of the Van der Waals potential and from how they are defined (i.e. the fact that they are considered proportional to the collisional broadening rates). Note that only if we calculate the rates as correctly defined in Eq. (12) and employ the Van der Waals potential for the atom-hydrogen interaction potential, will we find the rates underestimated by a factor of roughly two as one finds for the collisional broadening calculations (Papers I and IV).

\section{Conclusion}

The purpose of the present paper is to extend our theory to the calculation o $\mathrm{f}$ the depolarization and polarization transfer rates for $p(l=1), d(l=2)$ and $f(l=3)$ levels of complex atoms/ions allowing calculation of the rates for the upper levels of the lines of the multiplet 145 of neutral titanium Ti I. A great advantage of our method is that it is not specific for a given perturbed atom/ion and it is adaptable to any neutral and singly ionised atom which is in particular interesting for heavy atoms/ions. Of course, we can apply our theory to evaluate the depolarization and polarization transfer rates of more polarized lines of the second solar spectrum. The theory is a powerful tool to quickly calculate depolarization rates needed for the modelling of the second spectrum of the Sun.

\section{References}

Anstee, S. D., \& O’Mara, B. J. 1991, MNRAS, 253, 549

Anstee, S. D. 1992, Ph.D. Thesis, Univ. Queensland

Anstee, S. D., \& O'Mara, B. J. 1995, MNRAS, 276, 859

Anstee, S. D., O'Mara, B. J., \& Ross, J. E. 1997, MNRAS, 284, 202

Barklem, P. S., \& O'Mara, B. J. 1997, MNRAS, 290, 102

Barklem, P. S., \& O'Mara, B. J. 1998, MNRAS, 296, 1057

Barklem, P. S. 1998, Ph.D. Thesis, Univ. Queensland

Blum, K. 1981, Density Matrix Theory and Applications (New York: Plenum Press)

Bommier, V., Derouich, M., Landi Degl'Innocenti, E., Molodij, G., \& Sahal-Bréchot, S. A\&A, in press

Brink, D. M., \& Satchler, G. R. 1962, Angular Momentum (Oxford University Press)

Derouich, M. 2004, Ph.D. Thesis, Paris VII-Denis Diderot University, France

Derouich, M., Sahal-Bréchot, S., Barklem, P. S., \& B. J., O’Mara 2003a, A\&A, 404, 763 (Paper I)

Derouich, M., Sahal-Bréchot, S., \& Barklem, P. S. 2003b, A\&A, 409, 369 (Paper II)

Derouich, M., Sahal-Bréchot, S., \& Barklem, P. S. 2004a, A\&A, 414, 369 (Paper III)

Derouich, M., Sahal-Bréchot, S., \& Barklem, P. S. 2004b, A\&A, 426, 707 (Paper IV)

Gandorfer, A. 2000, The Second Sol. Spectrum: A high spectral resolution polarimetric survey of scattering polarization at the solar limb in graphical representation, Vol. 1: $4625 \AA$ to $6995 \AA$ (Hochschulverlag AG an der ETH Zurich)

Landi Degl'Innocenti, E. 1982, Sol. Phys., 79, 291

Manso Sainz, R., \& Landi Degl'Innocenti, E. 2002, A\&A, 394, 1093

Messiah, A. 1961, Mécanique Quantique (Paris: Dunod)

Roueff, E. 1974, J. Phys. B, 7, 185

Sahal-Bréchot, S. 1977, ApJ, 213, 887

Trujillo Bueno, J. 2003, The Generation and Transfer of Polarized Radiation in Stellar Atmospheres, in Stellar Atmosphere Modeling, ed. I. Hubeny, D. Mihalas, \& K. Werner, ASP Conf. Ser., 288, 551

Unsöld, A. L. 1927, Z. Phys., 43, 574

Unsöld, A. L. 1955, Physik der Stern Atmosphären (Zweite Auflage) 